\title{
Situation analysis for transport network development forecast in the MIX-PROSTOR system*
}

\author{
M. A. Bulyonkov, N. N. Filatkina
}

\begin{abstract}
The paper describes a system of scientific research automation applied to the forecasting of the development of Russian core transport network. The process of formation, evaluation and analysis of forecasted variants is considered. The variants can be combined, which allows for the implementation of the concept of the situation room that provides an intuitive user interface for decision-makers.
\end{abstract}

Keywords: scientific research automation, situation room, development of the transport system.

\section{Introduction}

The modern level of globalization and the growth of international trade have lead to a significant increase in the transportation of goods. Thanks to its geographical position, Russia has the potential to profit from the participation in international traffic, as a provider of transit services.

In order to realize this potential, the formation of Russian supporting transportation network should take into account the possible inclusion in the system of developing international transport corridors, which meets both the external and internal economic interests of the Russian Federation.

Forecasting the development of a single transport space includes a review of the problem on many levels - in fact, it is a system of hierarchically related tasks. These challenges require merging the methods and approaches developed in different areas of research. Undoubtedly, a key role is played by the construction of economic-mathematical models and methods of their calculation, but their use is not possible without a substantial information support. In our case, it is necessary to create an automated information management system with the support of GIS-technologies and scientific research automation.

There are a lot of software systems dealing with various transportation and infrastructure development problems on the municipal or even regional level. The largest, to our knowledge, is the PTV Vision @ $\cap$ VISUM system that allows for both operational and strategic planning of transportation in

${ }^{*}$ The work is a part of the Presidium of RAS project No. 31.3 "Integration role of large transportation project in special development". 
various conditions [1]. To emphasize the theoretical base and capacity of the system, the developers refer to the extensive communications of the company with fundamental research in the field of transport modeling methodology.

The article [2] discusses some ways to solve the problem of the modeling of transport development for an urban agglomeration on the basis of mathematical modeling methods of traffic flows. However, there are works that give a theoretical basis to the concept of the system (the transport system, in particular) potential and its usage for the system development forecast. The study of this potential is aimed at the improvement of complex systems which include interacting groups of people, mechanisms, etc [3].

Also, there are works devoted to the description of the transport network development model at the national level. For example, the main feature of the model in [4] is the absence of a single transportation system control centre, and the network dynamics is a result of self-organization due to the need for a good transportation and infrastructure development.

It is worth noting that when we talk about forecasting on the national level, it is often convenient to deal with transport corridors. Further details on the forming and development of transport corridors in Russia can be found in [5].

Our approach to the definition of Russian core transportation network is based on a group of economic-mathematical models of transport-economic balances (TEB) [6]. Transport-economic balances allow for describing and analyzing the relationships between the sectors of economics or regions under the conditions of competition between the different types of transport. In this formulation, it turns out that we set a forecast of the development of the parameters describing the services which can be provided by competing types of transport. Having this forecast set, we need to find out a variant of the development of the core transport network ensuring the rational interaction of its constituent types of transport. In other words, we solve the problem of forecasting by means of "what-if" simulation.

Our solution is based on the MIX system [7], jointly developed by Institute of Economics and Industrial Engineering of the Siberian Branch of the RAS, Institute of Informatics Systems of the Siberian Branch of the RAS, and Novosibirsk State University. The extension, called MIX-PROSTOR, allows for the quick building and testing of the various hypotheses of core transport network development, consistently adjusting the source data. Potential shipment volumes and different levels of tariffs may be set as an iteration over an interval with a specified step.

\section{Problem statement}

Our data model for transport network development forecast [8] includes the following entities: 
- product is a conditional group of goods for transportation, which are all produced and consumed goods;

- there are 5 job types of work related to the operation of the transport network: loading, unloading, transportation, transit, as well as transshipment from one type of transport to another for valid products and pairs of transport types;

- type of transport assumes indication of tariffs to perform each type of works for this type of transport.

- $h u b$ is the geographical point, for which possible operations on the processing of products of a certain type are defined. Since the hub is an operator, its maximum performance and coefficients for tariffs are to be specified for each type of work. There are different types of hubs: producers (primary loading points) and consumers (final unloading points);

- line is the basic unit of a transportation network connecting adjacent hubs. Each line is parameterized by length, capacity and tariffs for transportation for each type of transport and product. Lines are complemented by a sequence of geographic coordinates for rendering the corresponding path on the map.

\subsection{Model variables}

Let $I=\{i, \ldots\}$ be a set of all product types, $J=\{j, \tau, \ldots\}$ be a set of all transport types, and $R=\{r, s, \ldots\}$ be a set of all hubs. Then let us denote the following amounts of product $i$ processed at hub $r$ :

- $X_{i r}^{j}$ - accepted for loading on $j$

- $Y_{i r}^{j}$ - accepted for unloading on $j$

- $V_{i r}^{j \tau}$ - reloaded from $j$ on $\tau$

- $\bar{V}_{i r}^{\tau j}$ - reloaded from $\tau$ on $j$

- $Z_{i r}^{j}$ - transit on $j$

- $W_{i r s}^{j}$ - sent to adjacent hub $s$

- $\bar{W}_{i s r}^{j}$ - received from adjacent hub $r$

- $B_{i r}$ - the upper limit to be sent on all transport types

- $A_{i r}$ - the lower limit to be received on all transport types

Let us also denote

- $l_{r s}^{j}$ - the distance between adjacent $r$ and $s$ on transport $j$ 
- $\phi_{i r s}^{j}$ and $\bar{\phi}_{i r s}^{j}$ - specific with respect to the product amount unit, price for the transportation of $i$ on $j$ from $r$ to adjacent $s$ and back, respectively.

\subsection{Model constraints}

1. Constraint on the amount of the product $i$ generated and accepted for loading at the hub $r$ :

$$
\sum_{j} X_{i r}^{j} \leq B_{i r}
$$

2. Constraint on the amount of the product $i$ accepted for unloading at the hub $r$ :

$$
\sum_{j} Y_{i r}^{j} \leq A_{i r}
$$

3. The amount of the product $i$ that transits through $r$ on $j$ :

$$
Z_{i r}^{j}=\sum_{s} \bar{W}_{i s r}^{j}-Y_{i r}^{j}-\sum_{\tau} V_{i r}^{j \tau}
$$

4. The amount of the product reloaded from one transport to another:

$$
\sum_{\tau} V_{i r}^{j \tau}=X_{i r}^{j}+\sum_{s} \bar{W}_{i s r}^{j}+\sum_{\tau} V_{i r}^{\tau j}-Y_{i r}^{j}-\sum_{s} W_{i r s}^{j} .
$$

The objective function of the model is the minimum of expenses for the transportation of all products from hubs-producers to hubs-consumers. The functional can be represented as a sum of components corresponding to expenses from a particular transportation operation:

- processing of the product at hubs

- transportation of cargo per se.

Therefore,

$$
\sum_{i, r, j} c_{i}^{j} X_{i r}^{j}+\sum_{i, r, j} \bar{c}_{i}^{j} Y_{i r}^{j}+\sum_{i, r, j} c^{j} Z_{i r}^{j} \sum_{i, r, j \tau} c_{i r}^{j \tau} V_{i r}^{j \tau}+
$$




$$
+\sum_{i, r, \tau j} \bar{c}_{i r}^{\tau j} \bar{V}_{i r}^{\tau j}+\sum_{j, i, r s} \phi_{r s}^{j} l_{r s}^{j} W_{i r s}^{j}+\sum_{j, i, r s} \bar{\phi}_{s l}^{j} l_{r s}^{j} \bar{W}_{i s r}^{j} \rightarrow \min
$$

where the coefficients $c_{i}^{j}$ and $\bar{c}_{i r}^{\tau j}$ are specific with respect to the product amount unit, price for loading and unloading, respectively.

Thus we have formulated the problem of optimal transportation from hub-producers to hub-consumers. The solver for this problem is available and practically has been used for quite a long time [9]. Our aim is to provide an interactive graphical user interface and geo-informational support for the whole research process. We have identified the following tasks that need to be studied:

1. Editing the transport network. To do this, one must specify a list of products, transport types, relevant hubs and lines as well as their parameters, described above.

2. Modeling. When the transportation network is specified, the researcher may address the problems of forecasting such as "What will happen if tariffs on this particular line are halved?", or "What would be the impact on transportation if the production at this hub increased?", or "How much should the transportation tariffs on this line be reduced so that it becomes economically useful for transportation?" We must provide a user-friendly way for formulating possible changes of parameters and allow for comparisons of the results of the optimization solver for various sets of changes. In order to manipulate with the sets of changes, we have introduced the notion of series of variants that allows not only for changing a particular parameter value, but also for a range of values over which the value of a parameter is to be iterated with a specified step. This makes it possible for the researcher to run a series of tasks in the batch mode and select the best solution as the basis for additional changes on the next step of expert analysis.

3. Situation room. The specificity of the task prevents us from an unambiguous interpretation of the solution provided by the problem solver. The expert must interpret the results, analyze their property and decide which solution is the most promising. Moreover, the research result in this case is the correct formulation of the problem of the forecast that a researcher can make in the process of modeling various solutions. To be able to share an interesting forecast variant with other professionals who are not experts in the subject area, the concept of the situation room has been proposed. We will discuss these issues in more detail in the following section. 


\section{System architecture}

The MIX-PROSTOR system is an application of the MIX system developed to provide visual and geo-informational support of economic-mathematical modeling and analysis of various systems such as interaction of administrative regions, statistical data analysis of regions, transport network development forecast, forestry functioning visualization, etc.

Currently two applications are being actively developed: MIX-REGION and MIX-PROSTOR. In general, each application may have its own specific terminology, communication between informational objects, and utilize specialized software and additional interim storage. On the other hand, the visual and interactive support designed for different methods of economicmathematical models have a lot in common part in terms of their implementation and custom logic. Thus, different applications have similar visual components, such as components with map related information, tabular display of data or business graphics. For instance, in the task of traffic analysis the most natural way to select hubs or lines for setting their properties for forecasting is by pointing them on the map. On the other hand, in some cases it is more convenient to make adjustments to the forecast using the component of overall editing of the transport network. Thus, since the system provides several different ways to edit the information about the forecast, the components doing the editing must be synchronized. This interaction, including access to common data as well as generation and propagation of events, is realized by the system kernel MixEngine. The kernel consists of a domain-independent part and a collection of domain-specific functional elements: TransportGear, RegionGear, etc. Under this approach the access to all domain-specific knowledge is performed by corresponding functional elements.

One of the objectives of the initial design of MIX architecture was scalability with respect to two different dimensions. On the one hand, it is necessary to be able to add new models and new subject areas; on the other hand, we have to increase the visual capabilities of each subsystem.

The first dimension is provided by the structure of the system kernel and the ability to add new functional elements shown in the middle of Figure 1 as a set of interacting gears. Visual components are depicted as rectangles. The plug-in technology is used for component management; the list of dynamically loaded components is stored in a configuration file which can be tailored for a particular system application.

As each visual component has access to all kernel gears, one can develop a component that simultaneously operates on information from different subject areas, for example, concrete data available from the analysis of the forestry structure can be used to solve the problem of timber transportation.

Various combinations of the Visual component constitute a MIX ap- 


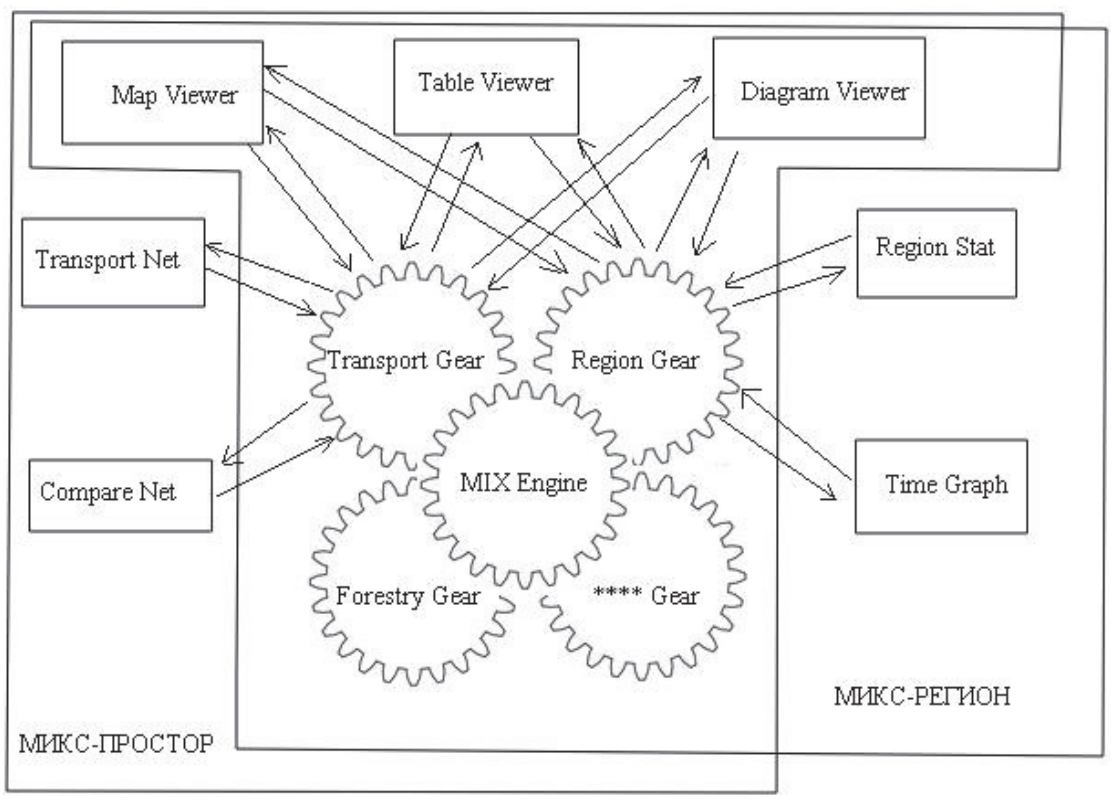

Figure 1. The structure of MIX

plication. In this paper we consider MIX-PROSTOR working with transport models. Event exchange and access to data in MIX-PROSTOR occur via TransportGear responsible for the transportation model. In particular, TransportGear initiates the utility, which solves the transportation optimization problem, and provides a data bridge between this utility and visualization components. Moreover, it allows for accessing the transport network, its modification and management of forecast variants with appropriate user interface and events.

\section{User scenario}

In the initial implementation of the MIX-PROSTOR we focused on the three user groups (roles):

- The administrator specifies the fundamental part of the transportation network, especially its geometry, as well as the general lists of product and transportation types.

- The expert builds some variants of development, in the problems of modeling and forecasting, by changing the quantitative network parameters for the given economic-mathematical model of a transportation task. 
- The decision maker experiments with combinations of predefined global situations and draws general conclusions about the development prospects, without going into details of quantitative parameters of the task.

However, in practice it has turned out that it does not make sense to separate the first two roles, since a variant of development may require not only a modification of quantitative parameters, but also an addition of new hubs and lines, as well as product and transport types. Therefore, our architecture provides specialized user interfaces and scenarios only for two roles. An expert in transportation modeling maintains the transportation network and formulates variants (situations) of its development, while an economist, who is not a specialist in mathematical models, or a decision maker considers the network on the macro level and investigates development scenarios by combination of prepared situations. Essentially, we present a situation room - an environment for analytics and decision making [10].

\section{Editing of network}

The MIX-PROSTOR provides a convenient interface for the creation and modification of a transport network. A typical view of the network editing panel is shown in Figure 2. The user may set the production and consumption of a selected product for each hub, where a positive number stands for production and a negative, for consumption. The last line shows the total, which must be greater or equal to zero for correct data.

\begin{tabular}{|c|c|}
\hline \multicolumn{2}{|c|}{$\begin{array}{l}\text { Настройка производства и } \\
\text { потребления ресурса } \\
\square \text { Показать все города }\end{array}$} \\
\hline Чзел & Значение \\
\hline Ляньюньган & -20 \\
\hline Новосибирск & -5 \\
\hline 中ранкфурт & +30 \\
\hline Ченджу & -5 \\
\hline Итого & 0 \\
\hline
\end{tabular}

Figure 2. Editing of the production and consumption of products

Other parameters of the transport network are treated similarly.

Currently, the MIX-PROSTOR system does not allow for several networks to be treated simultaneously. This limitation can be easily removed when needed. 


\section{Modeling}

The tariff and line capacity coefficients are the most flexible part of the network definition. We have introduced the notion of a variator that specifies a set of predicted values for some coefficient. A variator is a basic variation unit and consists of three parts: the initial value, the final value, and the number of steps. Thus a variator specifies a series of values of length equal to the number of steps. Starting from the initial value, each next value is obtained either by adding an increment or by multiplying by a factor such that the last value coincides with the final value of the variator. Depending on the type of the coefficient there are three types of variators:

1. Variators for the tariff of processing at a hub for a specific transport

2. Variators for the tariff of transportation along a selected line

3. Variators for the selected line capacity.

The user may create a variator with a dialog shown in Figure 2. Here the variator means that the capacity of the railroad line from Urumchi to Tashkent varies through values 50, 75, and 100 .

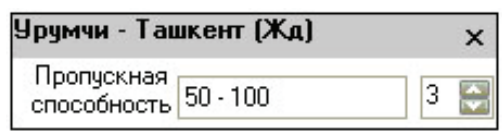

Figure 3. Editing of the variator

The forecast task is defined by a collection of variators, at most one for each coefficient. If a there is no coefficient for some parameter, the parameter is assumed to be unchanged. Since each variator specifies a series of values, a set of variators specifies a multi-dimensional matrix of all possible combinations of varied values. For each combination the optimization task must be solved. The MIX-PROSTOR system automatically generates and runs a batch of the invocation of the optimizing utility and displays the series of solutions to the user.

Currently, all variators are independent and vary asynchronously. In some cases, however, it would be good to synchronize some variators. Suppose, for instance, that we want to foresee what will happen if the tariffs on the Trans-Siberian railroad decrease by $10 \%, 20 \%$, and $30 \%$. Here we need to treat all the lines that constitute the railroad synchronously. A possible approach to this problem could be the introduction of a virtual object that aggregates a collection of lines and/or hubs and a subsequent variation of the parameters of all objects by a single variator.

To find interesting forecast variants the researcher needs a wide range of operations on variants. The system provides the ability to group variants 
and create a hierarchy of variants, extend the definition of a variant with variators from other variants, etc. If the research is conducted by a group of experts, variants may be shared.

\section{Visualizations of transportation problem solutions}

Currently we use two visual views to display a solution: a map and a table. The map representation is more natural since we are dealing with real geographical objects [11]. On the other hand, business graphics is more appropriate for the comparative analysis of numerical information.

As soon as a solution is obtained, the map and the table immediately display information related to all product types. This allows for getting "the big picture": see the network congestion, identify bottlenecks, etc. By filtering the results by the product type, the researcher can see the specific numerical values for the transportation of this product within the general transportation task.

These values are available in a table that contains all the lines participating in the solution with an indication of the starting and ending hubs for each line, the amount being transported and congestion with respect to the line capacity in percent. Using the sorting of table rows by any of these values, the researcher may easily see the lines where the workload exceeds the specified level. As for the map, the user may view the values related to a hub simply by hovering the mouse on the corresponding point on the map.

One of the most important characteristics of a solution for its evaluation in the context of forecasting is the degree of lines congestion. The system provides a special display mode that allows us to associate workload with colors: a gradient change from blue, which stands for zero workload, to red, which stands for full workload. In this way trouble spots can be quickly located. For example, in Figure 4 you can see a bottleneck on the chain of lines from Yekaterinburg to Novosibirsk.

The second display mode varies the width of a line according to the amount of a particular product type being transported along the line. Thus, the same solution will be displayed as shown in Figure 5, demonstrating that the bulk of traffic falls on the shipping routes through the Suez Canal, while the Trans-Kazakhstan line is used moderately.

The quality of comprehension can be greatly improved if both characteristics are displayed simultaneously, as presented in Figure 6. This figure demonstrates that the Trans-Siberian railway would not cope with the load if new products were transported along it since some of its lines (though not all) would be marked red as having a $100 \%$ congestion. In this situation, the Trans-Kazakhstan railway becomes a real competitor. 


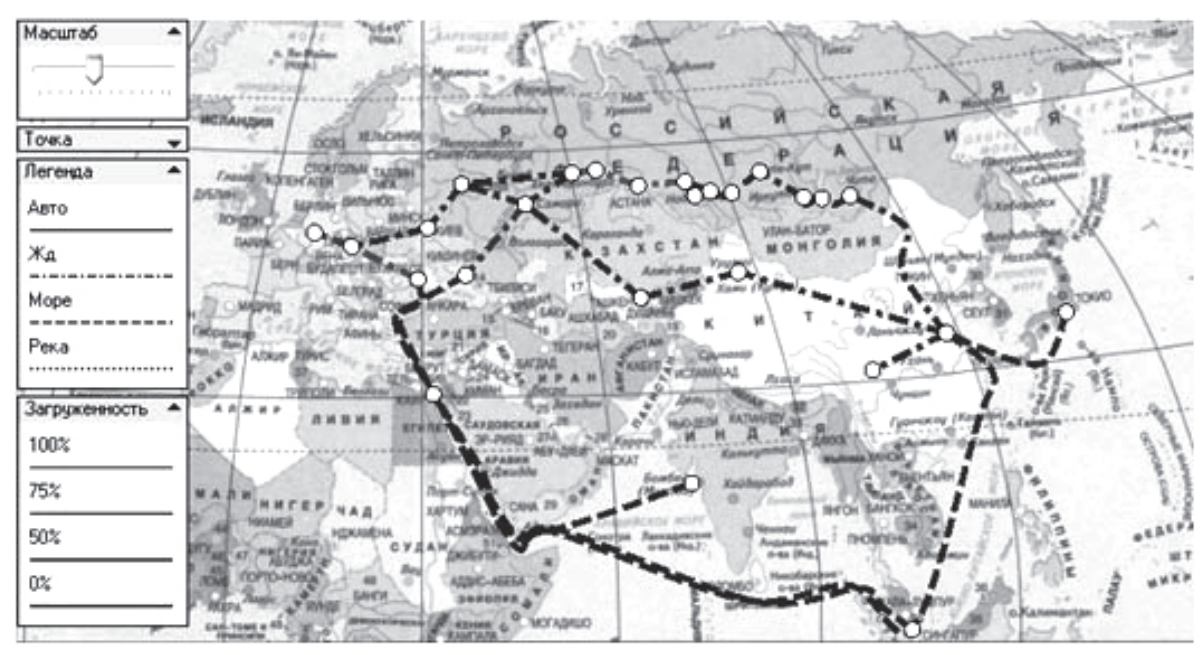

Figure 4. Display of relative line congestion

\section{Situation room}

When working on global development, the expert may wish to reduce the level of detail. We already did that on the level of the logical model of the transportation network by selecting only the hubs and lines significant for international transport flows. Next, the researcher may experiment with the model and identify a set of "basic situations", which are elementary changes of the model from the global point of view. For example, the expert selects all parts of the Trans-Siberian railway in one variant and sets the agreed changes that characterize the situation with tariff reduction along the railway. Then the resulting variant can be combined with changes in other parts of the global transportation system. A set of situations is the requisite of the situation room that allows for manipulating with changes on a higher logical level. In particular, transport corridors may be treated integrally.

For each basic situation, the expert provides a detailed verbal description of the economic sense of intended quantitative changes, which are specified by the variant. Then the decision maker selects a number of situations that may happen simultaneously in different parts of the world and obtains a forecast of their joint impact on the transportation network. The expert should guarantee that the situations are mutually consistent, which means that the corresponding underlying sets of parameters whose values are to be changed do not intersect. Thus each parameter is set unambiguously. If such a conflict does arise nonetheless, it is resolved by default in favor of the situation appearing later in the list.

The only difference between some situations is that they specify different values for the same parameter. In this case, these situations with a common 


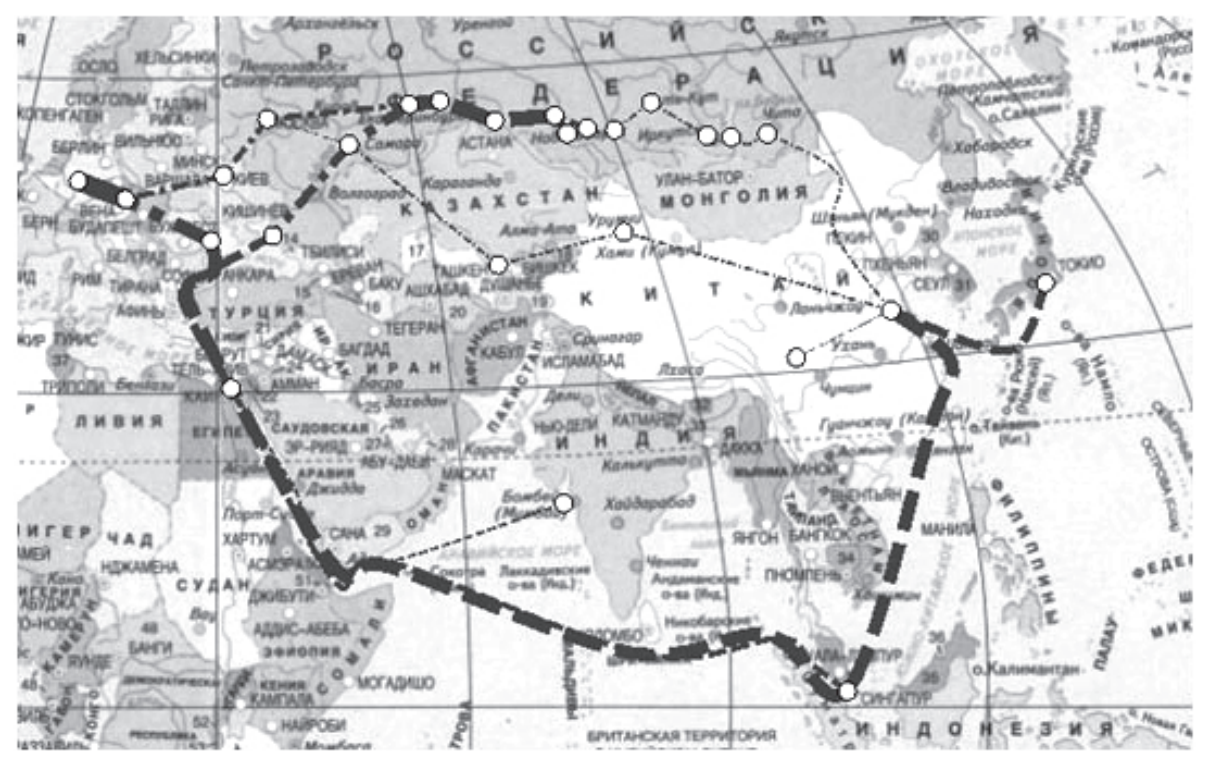

Figure 5. Display of transported amounts

description may be grouped, and the user can choose one of them. Consider, for example, three variants of tariff reduction on the Northern Sea Route: heavy $-50 \%$, moderate $-30 \%$, and small $-10 \%$. Implicitly we have the fourth variant as well, assuming unchanged tariff. In this case it is natural to treat these situations not as independent, but as alternatives of the same forecast. We have extended the data format of the situation room description and its visual representation, as shown in Figure 7.

The situation room dialog (Figure 8) is constructed dynamically based on a description file, which is created by an expert in the process of modeling. Dynamic generation makes it possible to easily extend the situation room and control modifications. Thus, we provide flexibility and customizability: the system can be easily adjusted to any other (including predicted and not really existing) transport network and another set of situations.

\section{Conclusion}

We have considered a research automation system as applied to the forecasting of the development of the Russian core transportation system. Crucial for a successful application of the system is the use of an interface adaptable to a specific group of users. In particular, the concept of the situation room raises the level of abstraction and, consequently, allows users to conduct meaningful experiments even without proper background in economic-mathematical modeling. 


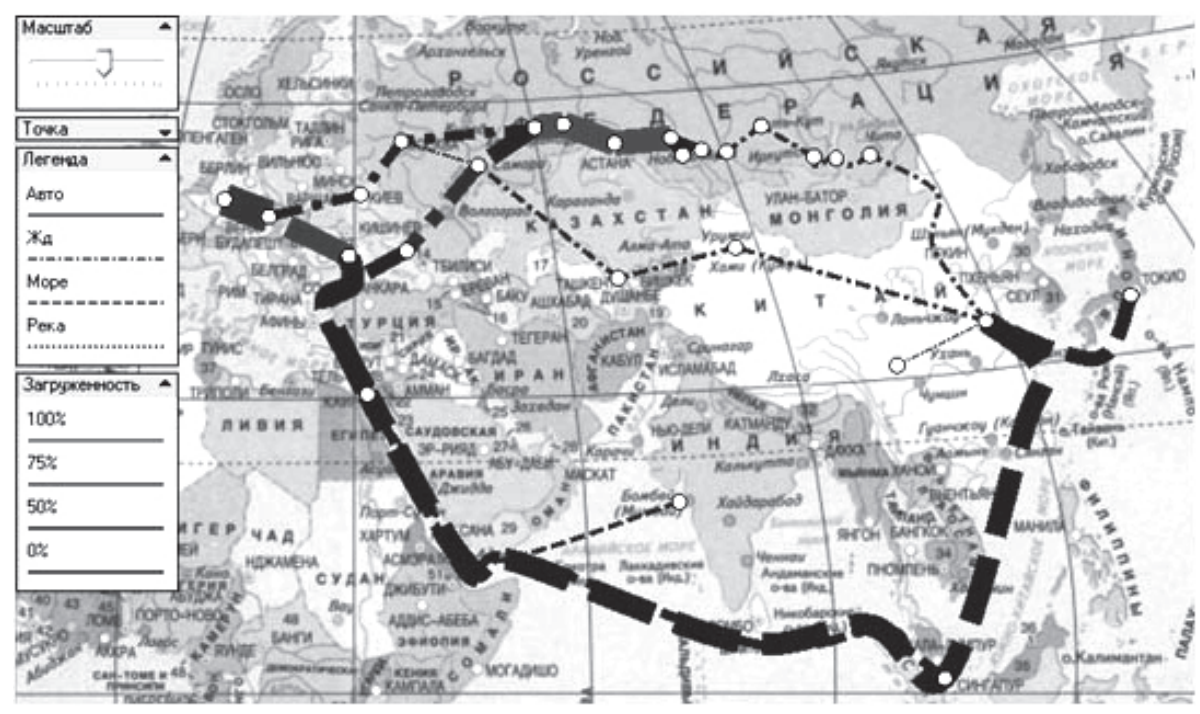

Figure 6. Joint display

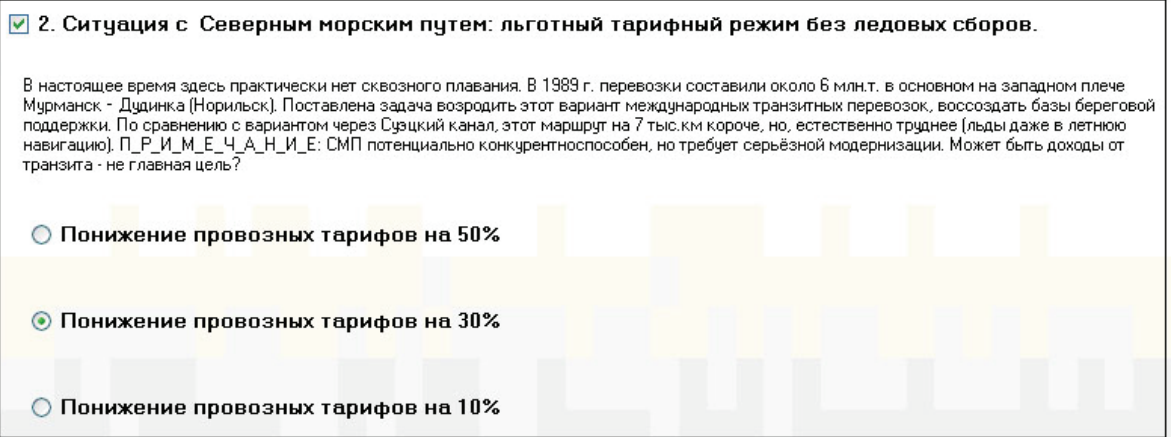

Figure 7. Alternative variants of a situation

We plan to enhance the system in a number of ways. First, methods of comparisons of different variants of development forecast might be very useful, especially when controlled by expert directives. Second, the development of the transportation system should be considered not in isolation, but in the context of the economic development trends and interaction of Russian regions. 


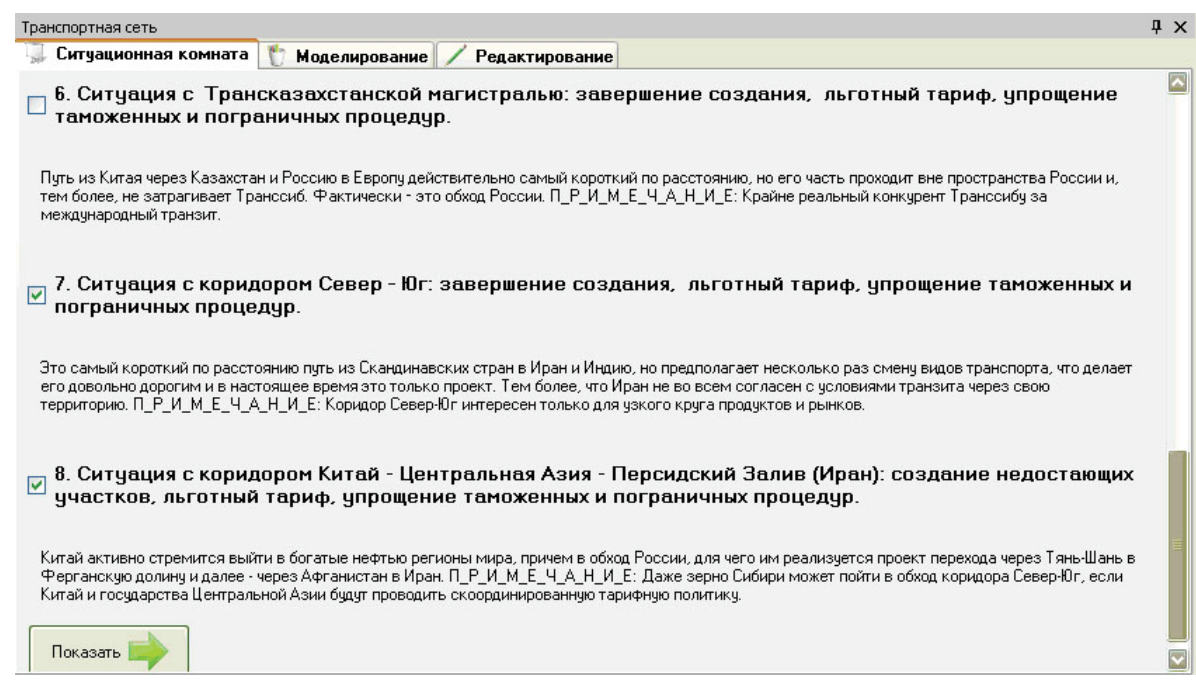

Figure 8. Situation room

\section{References}

[1] Shvetsov V.l., Prokhorov, A.V., Ilin I.V. Transport model in public administration // Scientific and Technical Statements of the SpBSPU. - 2009. N 5(85). - P. 20.

[2] Shvetsov V.I. Algorithms for traffic distribution // Automation and Telemechanics. - 2009. - N 10. - P. 148-157.

[3] Gada A.S., Lysenko I.V. Automating research tasks of potential of systems and the efficiency of their operation // Works of SPIIRAS. - 2012. - Iss. 22. - P. 260-281.

[4] Agapova G.I., Gavdaeva A.V., Stepantsov M.E. Modeling the dynamics of the development of railway networks. - 2011. - 12 p. - (Prep. / Keldysh IAM; N 73) URL: http://library.keldysh.ru/preprint.asp?id=2011-73

[5] Sergeev V.I., Goncharenko S.S., Prokofiev T.A. The formation of international transport corridors in the European regions of the North, Siberia and the Far East // Logistics Today. - 2011. - N 5. - P. 5-21.

[6] Vorobieva V.V., Malov V.Yu., Marusin V.V., Radchenko V.V. Predicting the formation of core transport network: tools of alternative calculations / / Siberia and the Far East in the long-term development of the integrated transport infrastructure of Eurasia / Ed.: S.N. Vasiliev [etc.]; IrSUR [et al.]. - IrkutskMoscow-Novosibirsk, 2011. - Ch. 4.15. - P. 516-522.

[7] Bulyonkov M.A., Karpan V.V., Malov V.Yu., Marusin V.V., Radchenko V.V. Conceptual issues of building Model Information Cartographic System (MIX) // Modeling of industrial and regional systems based on GIS and information 
technology: collection of scientific papers / Ed.: Yu.Sh. Blum, V.V. Radchenko - Novosibirsk: IEIE RAS, 2011. - P. 5-28.

[8] Vorobieva V.V., Malov V.Yu., Radchenko V.V., PotterM.V., Serebryannikov I.E. A prediction model of core transport network // Modeling of industrial and regional systems based on GIS and information technology: collection of scientific papers / Ed.: Blum Yu.Sh., Radchenko V.V. - Novosibirsk: IEIE RAS, 2011. - P. 68-96.

[9] Zabinyako G.I A program package for integer linear programming // Discrete Analysis and Operations Research. - 1999. - Ser. 2, 6:2. - P. 32-41.

[10] Raikov A.N. Situation room to support enterprise solutions // Open Systems. - 1999. - N 7-8. - P. 56-66.

[11] Turlapov V.E. Geographic Information Systems in Economics: Teaching Manual. - Nizhny Novgorod, Nizhny Novgorod branch of HSE, 2007. 
